\title{
The Bohr inequality for ordinary Dirichlet series
}

\author{
by \\ R. Balasubramanian (Chennai), B. Calado (Paris) \\ and H. QuefFÉLEC (Lille)
}

\begin{abstract}
We extend to the setting of Dirichlet series previous results of $\mathrm{H}$. Bohr for Taylor series in one variable, themselves generalized by V. I. Paulsen, G. Popescu and D. Singh or extended to several variables by L. Aizenberg, R. P. Boas and D. Khavinson. We show in particular that, if $f(s)=\sum_{n=1}^{\infty} a_{n} n^{-s}$ with $\|f\|_{\infty}:=\sup _{\Re s>0}|f(s)|<\infty$, then $\sum_{n=1}^{\infty}\left|a_{n}\right| n^{-2} \leq\|f\|_{\infty}$ and even slightly better, and $\sum_{n=1}^{\infty}\left|a_{n}\right| n^{-1 / 2} \leq C\|f\|_{\infty}, C$ being an absolute constant.
\end{abstract}

Introduction. A well-known inequality of $\mathrm{H}$. Bohr ([12]) states that if $f(z)=\sum_{n=0}^{\infty} a_{n} z^{n}$ is analytic and bounded in the open unit disc $\mathbb{D}$, and if $\|f\|_{\infty}:=\sup _{z \in \mathbb{D}}|f(z)|$, then

$$
\sum_{n=0}^{\infty}\left|a_{n}\right|\left(\frac{1}{3}\right)^{n} \leq\|f\|_{\infty} .
$$

Moreover, the value $1 / 3$ is sharp: if $\sum_{n=0}^{\infty}\left|a_{n}\right| r^{n} \leq\|f\|_{\infty}$ for each such $f$, then $r \leq 1 / 3$; and equality in (1) holds for constant functions only ([24]).

On the other hand, by the Cauchy-Schwarz inequality we have

$$
\sum_{n=0}^{\infty}\left|a_{n}\right| r^{n} \leq\left(\sum_{n=0}^{\infty}\left|a_{n}\right|^{2}\right)^{1 / 2}\left(\sum_{n=0}^{\infty} r^{2 n}\right)^{1 / 2} \leq C_{r}\|f\|_{\infty},
$$

with $C_{r}:=\left(1-r^{2}\right)^{-1 / 2}$ for each $r<1$ and each such $f$, since

$$
\left(\sum_{n=0}^{\infty}\left|a_{n}\right|^{2}\right)^{1 / 2}=\|f\|_{2} \leq\|f\|_{\infty} .
$$

Here $\|f\|_{2}$ is the norm of the function $f$ in the Hardy space $H^{2}([19])$. Recently, E. Bombieri and J. Bourgain ([14]) showed that the best possible $C_{r}$ in the inequality (2) is equivalent to $\left(1-r^{2}\right)^{-1 / 2}$ when $r \stackrel{\leq}{\rightarrow}$.

2000 Mathematics Subject Classification: 30B10, 30B50, 40A05, 42A45, 42B30, 11M41.

Key words and phrases: Dirichlet series, Bohr radius, Banach spaces of Dirichlet series, hypercontractivity. 
Note that (1) can be seen as another manifestation (see [4] or [5]) of the hypercontractivity properties of the Poisson kernel $\left(P_{r}\right)_{0 \leq r<1}$ since it states that this kernel, acting on $H^{\infty}$ by convolution, maps contractively $H^{\infty}$ to the space $\ell^{1}$ of summable sequences for $r \leq 1 / 3$, whereas (2) states that the same kernel always maps continuously $H^{\infty}$ to $\ell^{1}$ for $r<1$ (and of course not for $r=1$ ). For other hypercontractivity properties of the Poisson kernel $\left(P_{r}\right)_{0 \leq r<1}$, see for example [29].

Various extensions of Bohr's inequality have recently been proposed by different authors after P. G. Dixon used it in the construction of a Banach algebra satisfying the non-unital von Neumann inequality and non-isomorphic to a subalgebra of $\mathcal{L}(H)$, the algebra of bounded linear operators on the Hilbert space $H$ ([17]). For example, R. P. Boas and D. Khavinson ([9]) and L. Aizenberg ([1]) studied multidimensional analogues of (1) for the polydisc, ball or other domains, showing that the $n$-dimensional Bohr radius $K_{n}$ of the unit $n$-dimensional polydisc $\left\{\left(z_{1}, \ldots, z_{n}\right) \in \mathbb{C}^{n}: \forall j \in[1, n],\left|z_{j}\right|<1\right\}$ satisfies

$$
\frac{1}{3 \sqrt{n}}<K_{n}<\frac{2 \sqrt{\log n}}{\sqrt{n}}
$$

so that there is no Bohr phenomenon for power series in infinitely many variables.

Similarly, P. B. Djakov and M. S. Ramanujan studied the Bohr phenomenon when the space $\ell^{1}$ is replaced by $\ell^{p}, 1 \leq p \leq 2$ ([18]); C. Bénéteau, A. Dahlner, D. Khavinson and B. Korenblum studied the case of the Hardy spaces $H^{p}$ in one variable ([7] and [6]); and V. Paulsen, G. Popescu and D. Singh studied the following two restricted versions of (1) ([24]):

$$
\text { if } a_{0}=0, \text { then } \sum_{n=1}^{\infty}\left|a_{n}\right|\left(\frac{1}{\sqrt{2}}\right)^{n} \leq\|f\|_{\infty},
$$

and the value $1 / \sqrt{2}$ is optimal; and

$$
\text { if }\|f\|_{\infty}=1, \text { then }\left|a_{0}\right|^{2}+\sum_{n=1}^{\infty}\left|a_{n}\right|\left(\frac{1}{2}\right)^{n} \leq 1,
$$

and the value $1 / 2$ is optimal. See also [16] and [2].

$\mathrm{H}$. Bohr called attention to a formal connection between ordinary Dirichlet series and Taylor series in infinitely many variables ([12] or [25]). The aim of this work is precisely to perform a similar study for Banach spaces of ordinary Dirichlet series $\sum_{n=1}^{\infty} a_{n} n^{-s}$, where the multiplier $r^{n}$ is obviously replaced by $n^{-\sigma}$. This multiplier has also good hypercontractivity properties ([4] or [5]). More precisely, influenced by the recent survey by H. Helson [20], we will introduce the following terminology. 
Let $(\mathcal{E},\|\cdot\|)$ be a Banach space of Dirichlet series $f(s)=\sum_{n=1}^{\infty} a_{n} n^{-s}$ with a finite abscissa of convergence: $\sigma_{\mathrm{c}}(f)<\infty$. We attach to $\mathcal{E}$ two abscissas (reminiscent of the Bohr radii $1 / 3,1 / \sqrt{2}, \ldots$ ):

1) The isometric Bohr abscissa $\varrho_{1}(\mathcal{E})$, defined as the minimum of those $\sigma \geq 0$ such that $\sum_{n=1}^{\infty}\left|a_{n}\right| n^{-\sigma} \leq\|f\|$ for each $f \in \mathcal{E}$. We may have $\varrho_{1}(\mathcal{E})$ $=\infty$.

2) The isomorphic Bohr abscissa $\varrho(\mathcal{E})$, defined as the infimum of those $\sigma \geq 0$ such that $\sum_{n=1}^{\infty}\left|a_{n}\right| n^{-\sigma}<\infty$ for each $f \in \mathcal{E}$. Equivalently, by the closed graph theorem, $\varrho(\mathcal{E})$ is the infimum of those $\sigma \geq 0$ such that $\sum_{n=1}^{\infty}\left|a_{n}\right| n^{-\sigma} \leq C_{\sigma}\|f\|$ for each $f \in \mathcal{E}$, if one assumes that the linear forms $f \mapsto a_{n}(f)$ are uniformly bounded on $\mathcal{E}$. We will say that $\varrho(\mathcal{E})$ is attained if $\sum_{n=1}^{\infty}\left|a_{n}\right| n^{-\varrho(\mathcal{E})}<\infty$ for any $f \in \mathcal{E}$, which may happen due to the less brutal convergence of Dirichlet series compared to Taylor series.

We will denote as usual by $\Omega(n)$ the number of prime divisors of $n$, counted with their multiplicities. We will be interested in the following Banach spaces:

$\mathcal{H}^{\infty}$ is the space of bounded analytic functions $f$ on $\mathbb{C}_{+}:=\{s \in \mathbb{C}$ : $\Re s>0\}$ such that in some right half-plane $\mathbb{C}_{\sigma}:=\{s \in \mathbb{C}: \Re s>\sigma\}$ one can write $f$ as a convergent ordinary Dirichlet series $f(s)=\sum_{n=1}^{\infty} a_{n} n^{-s}$ (by a theorem of Bohr, [11] or [4] or [5], one can always take $\sigma=0)$.

For $k \geq 1, \mathcal{H}_{k}^{\infty}:=\left\{f(s)=\sum_{n=1}^{\infty} a_{n} n^{-s} \in \mathcal{H}^{\infty}: a_{n}=0\right.$ if $\left.\Omega(n)>k\right\}$.

For $1 \leq p<\infty, \mathcal{H}^{p}$ is the completion of the space of Dirichlet polynomials $P(s):=\sum_{n=1}^{N} a_{n} n^{-s}$ for the norm

$$
\|P\|_{\mathcal{H}^{p}}:=\left(\lim _{T \rightarrow \infty} \frac{1}{2 T} \int_{-T}^{T}|P(i t)|^{p} d t\right)^{1 / p}
$$

(the limit exists by the theory of almost periodic functions [13]).

$\mathcal{H}^{p}$ is isometric to the Hardy space $H^{p}\left(\mathbb{T}^{\infty}\right)$ of the infinite-dimensional torus, and if $f \in \mathcal{H}^{p}$, one can represent $f$ as an ordinary Dirichlet series $\sum_{n=1}^{\infty} a_{n} n^{-s}$ with $a_{n} \rightarrow 0$, so that $\sigma_{\mathrm{c}}(f) \leq 1$ (see [4] or [5] for more information on the spaces $\mathcal{H}^{p}$ ).

Using hypercontractivity properties of the Poisson kernel, F. Bayart ([4] or [5]) was able to prove that $\varrho\left(\mathcal{H}^{1}\right)=1 / 2$ (see also [20]). In fact, he proved that $\sum_{n=1}^{\infty}\left|a_{n}\right|^{2} / n^{\varepsilon}<\infty$ for each $\varepsilon>0$ and each $f \in \mathcal{H}^{1}$, so that by the Cauchy-Schwarz inequality,

$$
\sum_{n=1}^{\infty} \frac{\left|a_{n}\right|}{n^{1 / 2+\varepsilon}} \leq\left(\sum_{n=1}^{\infty} \frac{\left|a_{n}\right|^{2}}{n^{\varepsilon}}\right)^{1 / 2}\left(\sum_{n=1}^{\infty} \frac{1}{n^{1+\varepsilon}}\right)^{1 / 2}<\infty,
$$

and $\sigma_{\mathrm{c}}(f) \leq 1 / 2$.

Now, it follows from a celebrated and non-trivial theorem of H. F. Bohnenblust and E. Hille ([10]), answering a long-standing question of H. Bohr, 
that one also has $\varrho\left(\mathcal{H}^{\infty}\right)=1 / 2$, which, according to Helson's comments, is a little strange since $\mathcal{H}^{\infty}$ is a much smaller space than $\mathcal{H}^{1}$. One of our theorems shows that we can yet make a difference between the two spaces, in that $\varrho\left(\mathcal{H}^{\infty}\right)=1 / 2$ is attained, whereas $\varrho\left(\mathcal{H}^{1}\right)=1 / 2$ is not. The Bohr abscissa $\varrho\left(\mathcal{H}_{k}^{\infty}\right)$ is known to be equal to $1 / 2-1 /(2 k)([10])$; we will show that it is equally attained. The determination of the isometric Bohr abscissa $\varrho_{1}(\mathcal{E})$ is obviously more delicate, and whereas we are able to determine $\varrho(\mathcal{E})$ for the preceding spaces, we will content ourselves, apart from two exceptions, with estimates for $\varrho_{1}(\mathcal{E})$ (observe that $\varrho_{1}(\mathcal{E}) \geq \varrho(\mathcal{E})$ ). One of our theorems shows that $\varrho_{1}\left(\mathcal{H}^{\infty}\right)<\infty$, whereas there is no Bohr phenomenon for power series in infinitely many variables and Bohr's theory shows that $\mathcal{H}^{\infty}$ can be seen as a space of Taylor series on the infinite polydisc (see also [15]), so one might think that there is no such phenomenon for Dirichlet series. Comparing with [18], we shall also examine the effect of replacing the space $\ell^{1}$ by $\ell^{p}, 1 \leq p \leq 2$, and comparing with (3) and (4) ([24]), we shall examine the effect of taking $a_{1}=0$, or of replacing $\left|a_{1}\right|$ by $\left|a_{1}\right|^{2}$, without affecting the other terms. Accordingly, this work consists of two parts: the first is devoted to the study of the isomorphic Bohr abscissa, and the second to the more delicate study of the isometric Bohr abscissa.

We shall sometimes use the notation $E_{1} \ll_{(k)} E_{2}$ (resp. $E_{1} \gg_{(k)} E_{2}$ ) to say that $E_{1} \leq C_{(k)} E_{2}$ (resp. $E_{1} \geq C_{(k)} E_{2}$ ) where $C_{(k)}$ is an absolute positive constant (depending on $k$ only). Moreover, $\sum_{p}$ and $\sum_{p, q}$ will be summations over the prime numbers.

1. The isomorphic Bohr abscissa. The main theorem of this section is the following result:

THEOREM 1.1.

1) For $1 \leq q<\infty, \varrho\left(\mathcal{H}^{q}\right)=1 / 2$, but it is not attained.

2) $\varrho\left(\mathcal{H}^{\infty}\right)=1 / 2$, and it is attained.

Proof. As already mentioned, $\varrho\left(\mathcal{H}^{1}\right)=1 / 2$ follows from Bayart's result ([4] or [5]), and H. F. Bohnenblust and E. Hille proved that $\varrho\left(\mathcal{H}^{\infty}\right)=1 / 2$. So, for every $p \in[1, \infty], \varrho\left(\mathcal{H}^{p}\right)=1 / 2$ since $\mathcal{H}^{\infty} \subset \mathcal{H}^{p} \subset \mathcal{H}^{1}$. The novelty here is that only $\varrho\left(\mathcal{H}^{\infty}\right)$ is attained.

1) Recall that a sequence $\left(\lambda_{n}\right)_{n \geq 1}$ of complex numbers is completely multiplicative if $\lambda_{m n}=\lambda_{m} \lambda_{n}$ for all $m, n \in \mathbb{N}$. F. Bayart showed the following result ([4]):

Proposition 1.1. Let $p, q \geq 1$ with $p \leq q$ and $\left(\lambda_{n}\right)_{n \geq 1}$ be a completely multiplicative sequence such that $\lambda_{p_{k}} \leq \sqrt{p / q}$ for large $k$, where $p_{k}$ is the $k$ th prime number. Then $\left(\lambda_{n}\right)_{n \geq 1} \in \mathcal{M}\left(\mathcal{H}^{p}, \mathcal{H}^{q}\right)$, the set of multipliers from $\mathcal{H}^{p}$ to $\mathcal{H}^{q}$. 
Recall that a multiplier from $\mathcal{H}^{p}$ to $\mathcal{H}^{q}$ (resp. from $\mathcal{H}^{p}$ to $\ell^{q}$ ) is a sequence $\left(\mu_{n}\right)_{n \geq 1}$ such that $\sum_{n=1}^{\infty} \mu_{n} a_{n} n^{-s} \in \mathcal{H}^{q}$ (resp. $\left.\left(\mu_{n} a_{n}\right)_{n \geq 1} \in \ell^{q}\right)$ for each $f(s)=\sum_{n=1}^{\infty} a_{n} n^{-s}$ in $\mathcal{H}^{p}$.

Proposition 1.1 implies that for all $1 \leq q<\infty, \varrho\left(\mathcal{H}^{q}\right)$ is not attained. In fact take $p=2$ and $\left(\lambda_{n}\right)_{n \geq 1}$ the completely multiplicative sequence such that $\lambda_{p_{k}}=\sqrt{2 / q}$ for all $k$, where $p_{k}$ is the $k$ th prime number. Now, if $(1 / \sqrt{n})_{n \geq 1} \in \mathcal{M}\left(\mathcal{H}^{q}, \ell^{1}\right)$, we have $\left(\lambda_{n} / \sqrt{n}\right)_{n \geq 1} \in \mathcal{M}\left(\mathcal{H}^{2}, \ell^{1}\right)$ since if $\sum_{n=1}^{\infty} a_{n} n^{-s} \in \mathcal{H}^{2}$, then $\sum_{n=1}^{\infty} \lambda_{n} a_{n} n^{-s} \in \mathcal{H}^{q}$ and $\sum_{n=1}^{\infty}\left|\lambda_{n} a_{n} / \sqrt{n}\right|<\infty$. So, $\sum_{n=1}^{\infty}\left|\lambda_{n}\right|^{2} / n<\infty$, whence $\sum_{k=1}^{\infty}\left|\lambda_{p_{k}}\right|^{2} / p_{k}<\infty$, which contradicts the divergence of $\sum_{k=1}^{\infty} 1 / p_{k}$, since $\lambda_{p_{k}}$ is a positive constant independent of $k$.

2) We now show that $\varrho\left(\mathcal{H}^{\infty}\right)$ is attained. This will be a consequence of the following more general result:

Theorem 1.2. Set $\lambda(x):=\sqrt{\log x \log \log x}$ for $x \geq 3$ and $\lambda(x):=0$ for $x<3$. There exists a constant $\beta>0$ such that if $\left(\mu_{n}\right)_{n \geq 1}$ is a non-increasing sequence of non-negative real numbers, and if $\sum_{n=1}^{\infty} \mu_{n} e^{-c \lambda(n)} / \sqrt{n}<\infty$ for some $c<\beta$, then $\left(\mu_{n}\right)_{n \geq 1}$ is a multiplier from $\mathcal{H}^{\infty}$ to $\ell^{1}$.

Proof of Theorem 1.1. Theorem 1.2 implies Theorem 1.1 since we can take $\mu_{n}:=1 / \sqrt{n}$, or even $\mu_{n}:=e^{\delta \lambda(n)} / \sqrt{n}$ with $\delta<\beta$, since $\sum_{n=1}^{\infty} e^{-\varepsilon \lambda(n)} / n$ $<\infty$ for each $\varepsilon>0$.

Proof of Theorem 1.2. The proof will be conveniently split into three lemmas, the first of which is of independent interest.

Lemma 1.1. If $f(s)=\sum_{n=1}^{\infty} a_{n} n^{-s} \in \mathcal{H}^{\infty}$ and $x \geq 2$, then

$$
\left\|\sum_{n \leq x} a_{n} n^{-s}\right\|_{\infty} \leq C\|f\|_{\infty} \log x
$$

where $C>0$ is an absolute constant.

Proof. Without loss of generality, we can assume $x=N+1 / 2$, where $N$ is an integer. We use Perron's effective formula (see [28, p. 135]) to get, for each positive $T$,

$$
A(x):=\sum_{n \leq x} a_{n}=\frac{1}{2 i \pi} \int_{2-i T}^{2+i T} f(s) \frac{x^{s}}{s} d s+O\left(\frac{x^{2}}{T} \sum_{n=1}^{\infty} \frac{\left|a_{n}\right|}{n^{2}|\log (x / n)|}\right),
$$

where the $O$ is absolute. Observe moreover that $\left|a_{n}\right| \leq\|f\|_{\infty}$.

If $n>x$ then

$$
\left|\log \frac{x}{n}\right|=\log \frac{n}{x} \geq \log \frac{N+1}{N+1 / 2} \geq \frac{1}{4(N+1 / 2)},
$$

and if $n<x$ then

$$
\log \frac{x}{n} \geq \log \frac{N+1 / 2}{N} \geq \frac{1}{4 N}
$$


so that the error term is dominated by $x^{3}\|f\|_{\infty} / T=\|f\|_{\infty}$ if we choose $T:=x^{3}$. Now, let $0<\varepsilon<2$, and use the Cauchy formula to write

$$
\begin{aligned}
\int_{2-i T}^{2+i T} f(s) \frac{x^{s}}{s} d s= & \int_{\varepsilon-i T}^{\varepsilon+i T} f(s) \frac{x^{s}}{s} d s+\int_{\varepsilon}^{2} f(u+i T) \frac{x^{u+i T}}{u+i T} d u \\
& -\int_{\varepsilon}^{2} f(u-i T) \frac{x^{u-i T}}{u-i T} d u .
\end{aligned}
$$

The last two integrals are uniformly dominated by $\|f\|_{\infty} x^{2} / T=\|f\|_{\infty} / x$ (recall that $T=x^{3}$ ), so they can be forgotten. And for the first integral we have

$$
\begin{aligned}
\left|\int_{-T}^{T} f(\varepsilon+i t) \frac{x^{\varepsilon+i t}}{\varepsilon+i t} i d t\right| & \leq \int_{-T}^{T}\|f\|_{\infty} \frac{x^{\varepsilon}}{\sqrt{\varepsilon^{2}+t^{2}}} d t=2 x^{\varepsilon}\|f\|_{\infty} \int_{0}^{T / \varepsilon} \frac{d u}{\sqrt{u^{2}+1}} \\
& \leq 2 x^{\varepsilon}\|f\|_{\infty}\left(1+\int_{1}^{T / \varepsilon} \frac{d u}{u}\right) \ll x^{\varepsilon}\|f\|_{\infty} \log (T / \varepsilon) .
\end{aligned}
$$

We now set $\varepsilon:=1 / \log x$ to obtain

$$
|A(x)| \ll\|f\|_{\infty} \log \left(x^{3} \log x\right) \ll\|f\|_{\infty} \log x .
$$

Finally, for $s \in \mathbb{C}_{+}$, we apply what we have just seen to the function $f_{s}$ defined by $f_{s}(z):=f(s+z)$ to get

$$
\left|\sum_{n \leq x} a_{n} n^{-s}\right| \ll\left\|f_{s}\right\|_{\infty} \log x \ll\|f\|_{\infty} \log x
$$

which ends the proof of the lemma.

Lemma 1.2. There are numerical constants $\alpha, \beta>0$ such that

$$
\sum_{n=1}^{N}\left|a_{n}\right| \leq \alpha \sqrt{N} e^{-\beta \lambda(N)}\left\|\sum_{n=1}^{N} a_{n} n^{-s}\right\|_{\infty} .
$$

This is Theorem 4.3 in [22]. Recall that $\lambda(N)$ is as in Theorem 1.2.

Lemma 1.3. Let $N_{k}:=2^{k}$, and $f(s)=\sum_{n=1}^{\infty} a_{n} n^{-s} \in \mathcal{H}^{\infty}$. Then

$$
\sum_{N_{k} \leq n<N_{k+1}}\left|a_{n}\right| \ll \sqrt{N_{k}} e^{-\beta \lambda\left(N_{k}\right)}\|f\|_{\infty} \log N_{k},
$$

where $\beta$ is as in Lemma 1.2 .

Proof. We write $f_{k}(s)=\sum_{n=1}^{N_{k+1}} a_{n} n^{-s}$. Then we have

$$
\sum_{N_{k} \leq n<N_{k+1}}\left|a_{n}\right| \leq \sum_{n=1}^{N_{k+1}}\left|a_{n}\right| \leq \alpha \sqrt{N_{k+1}} e^{-\beta \lambda\left(N_{k+1}\right)}\left\|f_{k}\right\|_{\infty}
$$




$$
\begin{aligned}
& \ll \sqrt{N_{k+1}} e^{-\beta \lambda\left(N_{k+1}\right)}\|f\|_{\infty} \log N_{k+1} \quad \text { (by Lemma 1.1) } \\
& \ll \sqrt{N_{k}} e^{-\beta \lambda\left(N_{k}\right)}\|f\|_{\infty} \log N_{k+1} \quad\left(\text { since } \sqrt{N_{k+1}}=\sqrt{2} \sqrt{N_{k}}\right) \\
& \ll \sqrt{N_{k}} e^{-\beta \lambda\left(N_{k}\right)}\|f\|_{\infty} \log N_{k} \quad\left(\text { since } \log N_{k+1}=\log 2+\log N_{k}\right) .
\end{aligned}
$$

Now the end of the proof of Theorem 1.2 is easy: we may assume that $f(s)=\sum_{n=1}^{\infty} a_{n} n^{-s} \in \mathcal{H}^{\infty}$ and $\|f\|_{\infty} \leq 1$. For $\beta$ as in Lemma 1.2, we have

$$
\begin{aligned}
\sum_{n=1}^{\infty} \mu_{n}\left|a_{n}\right| & =\sum_{k=0}^{\infty}\left(\sum_{N_{k} \leq n<N_{k+1}} \mu_{n}\left|a_{n}\right|\right) \leq \sum_{k=0}^{\infty} \mu_{N_{k}}\left(\sum_{N_{k} \leq n<N_{k+1}}\left|a_{n}\right|\right) \\
& \ll \sum_{k=0}^{\infty} \mu_{N_{k}} \sqrt{N_{k}} e^{-\beta \lambda\left(N_{k}\right)} \log N_{k} \ll \sum_{k=0}^{\infty} \mu_{N_{k}} \sqrt{N_{k}} e^{-c \lambda\left(N_{k}\right)}
\end{aligned}
$$

(the logarithmic factor is absorbed in the exponential since $c<\beta$ ), whereas

$$
\begin{aligned}
\sum_{n=1}^{\infty} \frac{\mu_{n}}{\sqrt{n}} e^{-c \lambda(n)} & =\sum_{k=0}^{\infty}\left(\sum_{N_{k} \leq n<N_{k+1}} \frac{\mu_{n}}{\sqrt{n}} e^{-c \lambda(n)}\right) \\
& \geq \sum_{k=0}^{\infty} \frac{\mu_{N_{k+1}}}{\sqrt{N_{k+1}}} e^{-c \lambda\left(N_{k+1}\right)}\left(N_{k+1}-N_{k}\right) \\
& \gg \sum_{j=1}^{\infty} \mu_{N_{j}} \sqrt{N_{j}} e^{-c \lambda\left(N_{j}\right)}
\end{aligned}
$$

which proves that $\sum_{n=1}^{\infty} \mu_{n}\left|a_{n}\right|<\infty$ if $\sum_{n=1}^{\infty} \mu_{n} e^{-c \lambda(n)} / \sqrt{n}<\infty$.

REMARK 1.1. Observe that $\mu_{n}=1 / \sqrt{n}$ is decreasing and non-squaresummable, and is yet a multiplier from $\mathcal{H}^{\infty}$ to $\ell^{1}$. This is in marked contrast with the case of Taylor series: for example, if $H^{\infty}(\mathbb{D})$ denotes the space of analytic functions bounded in the unit disc, it is well known that the multipliers of $H^{\infty}(\mathbb{D})$ to $\ell^{1}$ are exactly the square-summable sequences; using the embedding $f \in H^{\infty}(\mathbb{D}) \mapsto f\left(2^{-s}\right) \in \mathcal{H}^{\infty}$, this shows that if $\left(\mu_{n}\right)_{n \geq 1} \in$ $\mathcal{M}\left(\mathcal{H}^{\infty}, \ell^{1}\right)$, then we must have $\sum_{n=1}^{\infty}\left|\mu_{2^{n}}\right|^{2}<\infty$, a fact which is already in Bayart's thesis ([4]).

We now examine the effect of replacing the space $\ell^{1}$ by $\ell^{p}$, as P. B. Djakov and M. S. Ramanujan did for Taylor series in [18]. We obtain the following result, which can be seen as a generalization of Theorem 1.1:

Theorem 1.3. Let $p \in[1,2]$. Then

1) For every $f(s)=\sum_{n=1}^{\infty} a_{n} n^{-s} \in \mathcal{H}^{\infty}, \sum_{n=1}^{\infty}\left|a_{n} n^{-\sigma}\right|^{p}<\infty$ if $\sigma \geq$ $1 / p-1 / 2=: \sigma_{0}$.

2) The value $\sigma_{0}$ is optimal: if $\sigma<\sigma_{0}$, we can find $f \in \mathcal{H}^{\infty}$ such that the sum in 1) is infinite. 
Proof. 1) We may assume that $\|f\|_{\infty}=1$. If $N_{k}=2^{k}$ for $k \geq 0$, we have

$$
\sum_{n=1}^{\infty}\left|a_{n} n^{-\sigma_{0}}\right|^{p}=\sum_{k=0}^{\infty}\left(\sum_{N_{k} \leq n<N_{k+1}}\left|a_{n} n^{-\sigma_{0}}\right|^{p}\right) \leq \sum_{k=0}^{\infty} N_{k}^{-p \sigma_{0}}\left(\sum_{n=1}^{N_{k+1}}\left|a_{n}\right|^{p}\right) .
$$

By Theorem 5.1 of [22],

$$
\left(\sum_{n=1}^{N_{k+1}}\left|a_{n}\right|^{p}\right)^{1 / p} \leq \alpha_{p} N_{k}^{1 / p-1 / 2} \exp \left(-\beta_{p} \lambda\left(N_{k}\right)\right)\left\|\sum_{n=1}^{N_{k+1}} a_{n} n^{-s}\right\|_{\infty},
$$

where $\alpha_{p}$ and $\beta_{p}$ are positive constants depending on $p$ only, and $\lambda$ is the function defined in Theorem 1.2. Using Lemma 1.1, we get

$$
\begin{aligned}
\sum_{n=1}^{\infty}\left|a_{n} n^{-\sigma_{0}}\right|^{p} & \ll p \sum_{k=0}^{\infty} N_{k}^{-p \sigma_{0}}\left[N_{k}^{1-p / 2}\left(\log N_{k}\right)^{p} \exp \left(-p \beta_{p} \lambda\left(N_{k}\right)\right)\right] \\
& =\sum_{k=0}^{\infty}\left(\log N_{k}\right)^{p} \exp \left(-p \beta_{p} \lambda\left(N_{k}\right)\right) \ll \sum_{k=0}^{\infty} k^{p} e^{-\sqrt{k}}<\infty .
\end{aligned}
$$

2) Let now $\sigma \in \mathbb{R}$ be such that $\sum_{n=1}^{\infty}\left|a_{n} n^{-\sigma}\right|^{p}<\infty$ for every $f(s)=$ $\sum_{n=1}^{\infty} a_{n} n^{-s} \in \mathcal{H}^{\infty}$. By the closed graph theorem, there exists a constant $C_{\sigma}$ such that for every $f(s)=\sum_{n=1}^{\infty} a_{n} n^{-s} \in \mathcal{H}^{\infty}$,

$$
\left(\sum_{n=1}^{\infty}\left|a_{n} n^{-\sigma}\right|^{p}\right)^{1 / p} \leq C_{\sigma}\|f\|_{\infty}
$$

So, for every Dirichlet polynomial $f(s)=\sum_{n=1}^{N} a_{n} n^{-s}$,

$$
\left(\sum_{n=1}^{N}\left|a_{n}\right|^{p}\right)^{1 / p} \leq C_{\sigma} N^{\sigma}\|f\|_{\infty}
$$

For $n \geq 2$, we shall denote by $P^{+}(n)$ the largest prime divisor of $n$. We will need the following lemma:

LEMMA 1.4. Let $y>1$ and $f(s)=\sum_{n=1}^{N} a_{n} n^{-s}$ be a Dirichlet polynomial such that $a_{n} \neq 0 \Rightarrow P^{+}(n) \leq y$. Let $f_{\omega}(s)=\sum_{n=1}^{N} \varepsilon_{n}(\omega) a_{n} n^{-s}$, $\omega \in \Omega$, where the $\varepsilon_{n}$ 's are independent Rademacher variables on the probability space $\Omega$. Then, with $E$ denoting expectation, we have

$$
E\left(\left\|f_{\omega}\right\|_{\infty}\right) \ll\left(\sum_{n=1}^{N}\left|a_{n}\right|^{2}\right)^{1 / 2} \sqrt{y \log \log N} .
$$

Proof. Let $P(z)=\sum a_{n} z_{1}^{\alpha_{1}} \cdots z_{r}^{\alpha_{r}}$, with $n=p_{1}^{\alpha_{1}} \cdots p_{r}^{\alpha_{r}}, p_{r} \leq y,\left|z_{j}\right|=1$, be the polynomial attached to $f$ by Bohr's theory, and let $P_{\omega}(z)=$ $\sum \varepsilon_{n}(\omega) a_{n} z_{1}^{\alpha_{1}} \cdots z_{r}^{\alpha_{r}}$ be the polynomial attached to $f_{\omega}$. Then Bohr's theory tells us that $\left\|P_{\omega}\right\|_{\infty}=\left\|f_{\omega}\right\|_{\infty}$ for each $\omega \in \Omega$. Moreover (see [21] or [25]), 
if $k$ is the number of variables $z_{j}$ in $P$ and $d=\max \left(\alpha_{1}+\cdots+\alpha_{r}\right)$ is its degree, we have

$$
E\left(\left\|P_{\omega}\right\|_{\infty}\right) \ll\left(\sum_{n=1}^{N}\left|a_{n}\right|^{2}\right)^{1 / 2} \sqrt{k \log d} .
$$

Here, $2^{\alpha_{1}+\cdots+\alpha_{r}} \leq p_{1}^{\alpha_{1}} \cdots p_{r}^{\alpha_{r}} \leq N$, so that $d \leq \log N / \log 2$, and if $p_{r}$ is the largest prime factor of any $n$ such that $a_{n} \neq 0$, we have $r \leq p_{r} \leq y$, so that $k \leq y$. The lemma clearly follows.

Let now $\varepsilon>0$. Using the preceding lemma with $y:=N^{\varepsilon}$, we can choose a sequence $\left(a_{n}\right)_{1 \leq n \leq N}$ such that $a_{n}:=0$ if $P^{+}(n)>N^{\varepsilon}$, and $a_{n}:= \pm 1$ otherwise, so as to have

$$
\left\|\sum_{n=1}^{N} a_{n} n^{-s}\right\|_{\infty} \ll \sqrt{N} \sqrt{N^{\varepsilon} \log \log N}=N^{1 / 2+\varepsilon / 2} \sqrt{\log \log N} .
$$

Using $(*)$ for this polynomial, we get

$$
\left(v_{N}\right)^{1 / p} \ll N^{1 / 2+\sigma+\varepsilon},
$$

where $v_{N}$ is the number of integers $n \in[1, N]$ with $P^{+}(n) \leq N^{\varepsilon}$. Since there exists $\delta_{\varepsilon}>0$ such that $v_{N}>\delta_{\varepsilon} N([28])$, we get $1 / 2+\sigma+\varepsilon \geq 1 / p$, that is, $\sigma \geq \sigma_{0}-\varepsilon$. This is true for all $\varepsilon>0$, so $\sigma \geq \sigma_{0}$, which ends the proof of the theorem.

We now turn to the subspace $\mathcal{H}_{k}^{\infty}$ of $\mathcal{H}^{\infty}$ and prove

Theorem 1.4. We have $\varrho\left(\mathcal{H}_{k}^{\infty}\right)=1 / 2-1 /(2 k)$, and it is attained. More precisely, for every $f(s)=\sum_{n=1}^{\infty} a_{n} n^{-s} \in \mathcal{H}_{k}^{\infty}$,

$$
\sum_{n=1}^{\infty} \frac{\left|a_{n}\right|(\log n)^{(k-1) / 2}}{n^{1 / 2-1 / 2 k}}<\infty
$$

Proof. First, observe that the case $k=1$ is given by Bohr's inequality stating that if $f(s)=\sum_{n=1}^{\infty} a_{n} n^{-s} \in \mathcal{H}^{\infty}$, then $\sum_{p}\left|a_{p}\right| \leq\|f\|_{\infty}$ ([25] or [26], see also Proposition 2.2 below). The value of $\varrho\left(\mathcal{H}_{k}^{\infty}\right)$ is already known ([10]); we prove that it is attained and even slightly better. Let $f(s)=$ $\sum_{n=1}^{\infty} a_{n} n^{-s} \in \mathcal{H}_{k}^{\infty}$ with $\left(a_{n}\right)_{n \geq 1}$ finitely supported, i.e. $a_{n}=0$ for $n$ large. We first assume that $k=2$. We attach to $f$ the function ( $p$ and $q$ running over the prime numbers)

$$
F(z):=a_{1}+\sum_{p} a_{p} z_{p}+\sum_{p, q: p \leq q} a_{p q} z_{p} z_{q}
$$

where $z:=\left(z_{p}\right)_{p \text { prime }}$ runs over the infinite polydisc $\mathbb{D}^{\infty}:=\prod_{p \text { prime }} \overline{\mathbb{D}}$, with $\overline{\mathbb{D}}:=\{z \in \mathbb{C}:|z| \leq 1\}$. It follows from Bohr's theory $([25])$ that $\|F\|_{\infty}:=$ $\sup _{\mathbb{D} \infty}|F|=\|f\|_{\infty}$. Bohr's inequality ([25] or [26]) says that $\sum_{p}\left|a_{p}\right| \leq$ $\|f\|_{\infty}$, and similarly, since 


$$
\sum_{p, q: p \leq q} a_{p q} z_{p} z_{q}=\frac{1}{2 \pi} \int_{0}^{2 \pi} F\left(e^{i \theta} z\right) e^{-2 i \theta} d \theta
$$

we have

$$
\left|\sum_{p, q: p \leq q} a_{p q} z_{p} z_{q}\right| \leq\|F\|_{\infty}=\|f\|_{\infty} .
$$

Using a polarization inequality due to L. Harris ([25, p. 50]) we get, if $\left(w_{q}\right)_{q \text { prime }}$ runs over the infinite polydisc (the inequality would be obvious with the constant 2 instead of $3 \sqrt{3} / 4)$ :

$$
\left|\sum_{p, q: p \leq q} a_{p q} z_{p} w_{q}\right| \leq \frac{3 \sqrt{3}}{4}\|f\|_{\infty} .
$$

Optimizing with respect to the $z_{p}$ 's, we get

$$
\sum_{p}\left|\sum_{q: q \geq p} a_{p q} w_{q}\right| \leq \frac{3 \sqrt{3}}{4}\|f\|_{\infty} .
$$

We now take for the $w_{q}$ 's independent complex Steinhaus random variables taking values of modulus 1 with the uniform distribution, integrate with respect to the $w_{q}$ 's and use the Khinchin inequalities $([27])$ to get

$$
\sum_{p}\left(\sum_{q: q \geq p}\left|a_{p q}\right|^{2}\right)^{1 / 2}=: \sum_{p} \lambda_{p} \leq \frac{3 \sqrt{3}}{2 \sqrt{\pi}}\|f\|_{\infty} .
$$

Similarly,

$$
\sum_{q}\left(\sum_{p: p \leq q}\left|a_{p q}\right|^{2}\right)^{1 / 2}=: \sum_{q} \mu_{q} \leq \frac{3 \sqrt{3}}{2 \sqrt{\pi}}\|f\|_{\infty} .
$$

Now, we have to show that

$$
\sum_{p, q: p \leq q} \frac{\left|a_{p q}\right|(\log (p q))^{1 / 2}}{(p q)^{1 / 4}} \ll\|f\|_{\infty},
$$

since we already know that $\left|a_{1}\right| \leq\|f\|_{\infty}$ and

$$
\sum_{p} \frac{\left|a_{p}\right|(\log p)^{1 / 2}}{p^{1 / 4}} \ll \sum_{p}\left|a_{p}\right| \leq\|f\|_{\infty} .
$$

Using the Cauchy-Schwarz inequality in the second line below, the fact that

$$
\sum_{p \leq x} p^{-\alpha} \underset{x \rightarrow \infty}{\sim} \frac{x^{1-\alpha}}{\log x} \quad \text { for } 0<\alpha<1
$$

(see [28]) in the third line, and (6) for the last inequality, we have 


$$
\begin{aligned}
\sum_{p, q: p \leq q} \frac{\left|a_{p q}\right|(\log (p q))^{1 / 2}}{(p q)^{1 / 4}} & \ll \sum_{q} \frac{(\log q)^{1 / 2}}{q^{1 / 4}}\left(\sum_{p: p \leq q} \frac{\left|a_{p q}\right|}{p^{1 / 4}}\right) \\
& \leq \sum_{q} \frac{(\log q)^{1 / 2}}{q^{1 / 4}}\left(\sum_{p: p \leq q}\left|a_{p q}\right|^{2}\right)^{1 / 2}\left(\sum_{p: p \leq q} \frac{1}{p^{1 / 2}}\right)^{1 / 2} \\
& \ll \sum_{q} \frac{(\log q)^{1 / 2}}{q^{1 / 4}} \mu_{q}\left(\frac{\sqrt{q}}{\log q}\right)^{1 / 2}=\sum_{q} \mu_{q} \ll\|f\|_{\infty} .
\end{aligned}
$$

This settles the case $k=2$ for the finitely supported case. We explain how to conclude in the general case after dealing with the case $k \geq 3$.

We now assume that $k \geq 3$. If $p_{l}$ is the $l$ th prime number for $l \in \mathbb{N}$, we have

$$
\begin{aligned}
f(s)= & a_{1}+\sum_{j_{1} \geq 1} a_{p_{j_{1}}} p_{j_{1}}^{-s}+\sum_{\substack{j_{1}, j_{2} \\
1 \leq j_{1} \leq j_{2}}} a_{p_{j_{1}} p_{j_{2}}}\left(p_{j_{1}} p_{j_{2}}\right)^{-s} \\
& +\cdots+\sum_{\substack{j_{1}, \ldots, j_{k} \\
1 \leq j_{1} \leq \cdots \leq j_{k}}} a_{p_{j_{1}} \cdots p_{j_{k}}}\left(p_{j_{1}} \cdots p_{j_{k}}\right)^{-s} \\
= & g(s)+\sum_{\substack{j_{1}, \ldots, j_{k} \\
1 \leq j_{1} \leq \cdots \leq j_{k}}} a_{p_{j_{1}} \cdots p_{j_{k}}}\left(p_{j_{1}} \cdots p_{j_{k}}\right)^{-s},
\end{aligned}
$$

with $g \in \mathcal{H}_{k-1}^{\infty}$. As before we attach to $f$ the function

$$
\begin{aligned}
F(z):= & a_{1}+\sum_{j_{1} \geq 1} a_{p_{j_{1}}} z_{p_{j_{1}}}+\sum_{\substack{j_{1}, j_{2} \\
1 \leq j_{1} \leq j_{2}}} a_{p_{j_{1}} p_{j_{2}}} z_{p_{j_{1}}} z_{p_{j_{2}}} \\
& +\cdots+\sum_{\substack{j_{1}, \ldots, j_{k} \\
1 \leq j_{1} \leq \cdots \leq j_{k}}} a_{p_{j_{1}} \ldots p_{j_{k}}} z_{p_{j_{1}}} \cdots z_{p_{j_{k}}} \\
= & G(z)+\sum_{\substack{j_{1}, \ldots, j_{k} \\
1 \leq j_{1} \leq \cdots \leq j_{k}}} a_{p_{j_{1}} \ldots p_{j_{r}}} z_{p_{j_{1}}} \cdots z_{p_{j_{k}}},
\end{aligned}
$$

where $z:=\left(z_{p}\right)_{p \text { prime }}$ runs over the infinite polydisc $\mathbb{D}^{\infty}:=\prod_{p \text { prime }} \overline{\mathbb{D}}$, and $G$ is the function attached to $g$ in the same way. For $l \in[1, k]$, we have

$$
\sum_{\substack{j_{1}, \ldots, j_{l} \\ 1 \leq j_{1} \leq \cdots \leq j_{l}}} a_{p_{j_{1}} \ldots p_{j_{l}}} z_{p_{j_{1}}} \cdots z_{p_{j_{l}}}=\frac{1}{2 \pi} \int_{0}^{2 \pi} F\left(e^{i \theta} z\right) e^{-l i \theta} d \theta
$$

so that

$$
\left|\sum_{\substack{j_{1}, \ldots, j_{l} \\ 1 \leq j_{1} \leq \cdots \leq j_{l}}} a_{p_{j_{1}} \ldots p_{j_{l}}} z_{p_{j_{1}}} \ldots z_{p_{j_{l}}}\right| \leq\|F\|_{\infty}:=\sup _{\mathbb{D} \infty}|F|=\|f\|_{\infty}
$$


(the last equality follows from Bohr's theory [25]) and by immediate induction we only need to show that

$$
\sum_{\substack{j_{1}, \ldots, j_{k} \\ 1 \leq j_{1} \leq \cdots \leq j_{k}}} \frac{\left|a_{p_{j_{1}} \cdots p_{j_{k}}}\right|\left(\log \left(p_{j_{1}} \cdots p_{j_{k}}\right)\right)^{k \tau}}{\left(p_{j_{1}} \cdots p_{j_{k}}\right)^{\tau}} \ll_{k}\|f\|_{\infty}
$$

with $\tau:=1 / 2-1 /(2 k)$. Using (7) for $l=k$ and Harris's polarization inequality $([25$, p. 50$])$ we get, if $\left(w_{q}^{(2)}\right)_{q \text { prime }}, \ldots,\left(w_{q}^{(k)}\right)_{q \text { prime }}$ run over the infinite polydisc,

$$
\begin{aligned}
\left|\sum_{j_{1} \geq 1} z_{p_{j_{1}}}\left(\sum_{\substack{j_{2}, \ldots, j_{k} \\
j_{1} \leq j_{2} \leq \cdots \leq j_{k}}} a_{p_{j_{1}} p_{j_{2}} \ldots p_{j_{k}}} w_{p_{j_{2}}}^{(2)} \cdots w_{p_{j_{k}}}^{(k)}\right)\right| \\
=\left|\sum_{\substack{j_{1}, j_{2} \ldots, j_{k} \\
1 \leq j_{1} \leq j_{2} \leq \cdots \leq j_{k}}} a_{p_{j_{1}} p_{j_{2}} \cdots p_{j_{k}}} z_{p_{j_{1}}} w_{p_{j_{2}}}^{(2)} \cdots w_{p_{j_{k}}}^{(k)}\right| \\
\leq c_{k}\|f\|_{\infty},
\end{aligned}
$$

where

$$
c_{k}:=\frac{k^{k / 2}(k+1)^{(k+1) / 2}}{2^{k} k !} .
$$

Optimizing with respect to the $z_{p_{j_{1}}}$ 's gives

$$
\sum_{j_{1} \geq 1}\left|\sum_{\substack{j_{2}, \ldots, j_{k} \\ j_{1} \leq j_{2} \leq \cdots \leq j_{k}}} a_{p_{j_{1}} p_{j_{2}} \cdots p_{j_{k}}} w_{p_{j_{2}}}^{(2)} \cdots w_{p_{j_{k}}}^{(k)}\right| \leq c_{k}\|f\|_{\infty} .
$$

We now take for the $w_{q}^{(l)}$ 's, $2 \leq l \leq k$, independent complex Steinhaus random variables taking values of modulus 1 with the uniform distribution, integrate with respect to the $w_{q}^{(l)}$, s and use the Khinchin inequalities ([25]) to get

$$
\sum_{j_{1} \geq 1}\left(\sum_{\substack{j_{2}, \ldots, j_{k} \\ j_{1} \leq j_{2} \leq \cdots \leq j_{k}}}\left|a_{p_{j_{1}} p_{j_{2}} \ldots p_{j_{k}}}\right|^{2}\right)^{1 / 2}=: \sum_{j_{1} \geq 1} \lambda_{1}\left(p_{j_{1}}\right) \leq C_{k}\|f\|_{\infty}
$$

where $C_{k}$ is a positive constant depending on $k$ only, which could be taken equal to $c_{k}(2 / \sqrt{\pi})^{k-1}$. Hence, if $j_{k}$ is fixed, then

$$
\begin{gathered}
\sum_{\substack{j_{1}, \ldots, j_{k-1} \\
1 \leq j_{1} \leq \cdots \leq j_{k-1}}} \frac{\left|a_{p_{j_{1}} \ldots p_{j_{k}}}\right|\left(\log \left(p_{j_{1}} \cdots p_{j_{k}}\right)\right)^{k \tau}}{\left(p_{j_{1}} \cdots p_{j_{k}}\right)^{\tau}} \\
\ll_{k} \frac{\left(\log p_{j_{k}}\right)^{k \tau}}{p_{j_{k}}^{\tau}}\left(\sum_{\substack{j_{1}, \ldots, j_{k-1} \\
1 \leq j_{1} \leq \cdots \leq j_{k-1}}} \frac{\left|a_{p_{j_{1}} \cdots p_{j_{k}}}\right|}{\left(p_{j_{1}} \cdots p_{j_{k-1}}\right)^{\tau}}\right)
\end{gathered}
$$




$$
\begin{aligned}
& \leq \frac{\left(\log p_{j_{k}}\right)^{k \tau}}{p_{j_{k}}^{\tau}}\left(\sum_{\substack{j_{1}, \ldots, j_{k-1} \\
1 \leq j_{1} \leq \cdots \leq j_{k}}} \frac{\left|a_{p_{j_{1}} \cdots p_{j_{k}}}\right|^{2}}{\left(p_{j_{1}} \cdots p_{j_{k-1}}\right)^{2 \tau}}\right)^{1 / 2} \\
& \quad \times\left(\sum_{\substack{j_{1}, \ldots, j_{k-1} \\
1 \leq j_{1} \leq \cdots \leq j_{k}}} \frac{1}{\left(p_{j_{1}} \cdots p_{j_{k-1}}\right)^{2 \tau}}\right)^{1 / 2} \\
& \leq \frac{\left(\log p_{j_{k}}\right)^{k \tau}}{p_{j_{k}}^{\tau} \lambda_{k}\left(p_{j_{k}}\right)\left(\left[\sum_{p: p \leq p_{j_{k}}} p^{-2 \tau}\right]^{k-1}\right)^{1 / 2}} \\
& \ll k \frac{\left(\log p_{j_{k}}\right)^{k \tau}}{p_{j_{k}}^{\tau}} \lambda_{k}\left(p_{j_{k}}\right)\left(\left[\frac{p_{j_{k}}^{1-2 \tau}}{\log p_{j_{k}}}\right]^{k-1}\right)^{1 / 2},
\end{aligned}
$$

which is $\lambda_{k}\left(p_{j_{k}}\right)$ since $\tau=1 / 2-1 /(2 k)$. Finally,

$$
\sum_{\substack{j_{1}, \ldots, j_{k} \\ 1 \leq j_{1} \leq \cdots \leq j_{k}}} \frac{\left|a_{p_{j_{1}} \cdots p_{j_{k}}}\right|\left(\log \left(p_{j_{1}} \cdots p_{j_{k}}\right)\right)^{k \tau}}{\left(p_{j_{1}} \cdots p_{j_{k}}\right)^{\tau}} \ll_{k} \sum_{j_{k} \geq 1} \lambda_{k}\left(p_{j_{k}}\right) \leq C_{k}\|f\|_{\infty}
$$

which settles the case $k \geq 3$ for the finitely supported case.

Let now $f(s)=\sum_{n=1}^{\infty} a_{n} n^{-s} \in \mathcal{H}_{k}^{\infty}$. We use Bohr's theorem which states that for $\varepsilon>0$ the series $\sum_{n=1}^{\infty} a_{n} n^{-\varepsilon} n^{-s}$ is uniformly convergent in $\mathbb{C}_{+}\left([11]\right.$, [4] or [5]), so that we can find an $N_{\varepsilon}>\varepsilon^{-1}$ such that $\left\|\sum_{n=N_{\varepsilon}+1}^{\infty} a_{n} n^{-\varepsilon} n^{-s}\right\|_{\infty} \leq \varepsilon$, and therefore $\left\|\sum_{n=1}^{N_{\varepsilon}} a_{n} n^{-\varepsilon} n^{-s}\right\|_{\infty} \leq\|f\|_{\infty}+\varepsilon$. Let $N$ be an integer and $\varepsilon>0$ be such that $N_{\varepsilon} \geq N$. The finitely supported case gives

$$
\sum_{n=1}^{N} \frac{\left|a_{n} n^{-\varepsilon}\right|(\log n)^{k \tau}}{n^{\tau}} \leq \sum_{n=1}^{N_{\varepsilon}} \frac{\left|a_{n} n^{-\varepsilon}\right|(\log n)^{k \tau}}{n^{\tau}} \leq A_{k}\left(\|f\|_{\infty}+\varepsilon\right),
$$

where $A_{k}$ is a positive constant depending on $k$ only, and letting $\varepsilon \rightarrow 0$ we get

$$
\sum_{n=1}^{N} \frac{\left|a_{n}\right|(\log n)^{k \tau}}{n^{\tau}} \leq A_{k}\|f\|_{\infty}
$$

This holds for every integer $N$, so

$$
\sum_{n=1}^{\infty} \frac{\left|a_{n}\right|(\log n)^{k \tau}}{n^{\tau}} \leq A_{k}\|f\|_{\infty}
$$

which ends the proof of Theorem 1.4.

We end this section with a result similar to Theorem 1.3 for the space $\mathcal{H}_{k}^{\infty}$ : 
Theorem 1.5. Let $p \in[1,2]$. Then:

1) For every $f(s)=\sum_{n=1}^{\infty} a_{n} n^{-s} \in \mathcal{H}_{k}^{\infty}, \sum_{n=1}^{\infty}\left|a_{n} n^{-\sigma}\right|^{p}<\infty$ if $\sigma \geq$ $\max (1 / p-1 / 2-1 /(2 k), 0)=: \sigma_{0}$.

2) The value $\sigma_{0}$ is optimal: if $\sigma<\sigma_{0}$, we can find $f \in \mathcal{H}_{k}^{\infty}$ such that the sum in 1) is infinite.

We do not give the proof of this result, which will appear in the second author's thesis.

2. The isometric Bohr abscissa. We begin with a lemma, analogous to a lemma of F. W. Wiener for Taylor series ([24]), and which will play an important role. Indeed, F. W. Wiener gave one of the simplest proofs of Bohr's inequality (1) (see [12]), observing that if $f(z)=\sum_{n=0}^{\infty} a_{n} z^{n} \in H^{\infty}$ with $\|f\|_{\infty}=1$, then

$$
\forall n \geq 1, \quad\left|a_{n}\right| \leq 1-\left|a_{0}\right|^{2} .
$$

We now give two different proofs of the following lemma, the first one operator-theoretic and an adaptation of the proof of [24], the second one more analytic.

Lemma 2.1. Let $f(s)=\sum_{n=1}^{\infty} a_{n} n^{-s} \in \mathcal{H}^{\infty}$ with $\|f\|_{\infty}=1$. Then

$$
\forall n \geq 2, \quad\left|a_{n}\right| \leq 1-\left|a_{1}\right|^{2} .
$$

First proof of Lemma 2.1. Let $\mathcal{H}^{2}$ be the Hilbert space of Dirichlet series $f(s)=\sum_{n=1}^{\infty} a_{n} n^{-s}$ with square-summable coefficients: $\|f\|_{2}^{2}:=\sum_{n=1}^{\infty}\left|a_{n}\right|^{2}$ $<\infty$. It is known ([4] or [5]) that the $n^{-s}, n \geq 1$, form an orthonormal basis of $\mathcal{H}^{2}$ (obvious) and that $\mathcal{H}^{\infty}$ is isometrically equal to the space of multipliers of $\mathcal{H}^{2}$ (non-obvious) in the sense that

$$
f \in \mathcal{H}^{2}, g \in \mathcal{H}^{\infty} \Rightarrow f g \in \mathcal{H}^{2} \text {, and moreover }\|g\|_{\infty}=\sup _{\|f\|_{2} \leq 1}\|f g\|_{2}
$$

(and conversely: if $f g \in \mathcal{H}^{2}$ for each $f \in \mathcal{H}^{2}$, then $g \in \mathcal{H}^{\infty}$ ).

Now, fix $q \geq 2$, let $P$ be the orthogonal projection of $\mathcal{H}^{2}$ onto $M:=$ $\operatorname{span}\left(1, q^{-s}\right)$, and $T: M \rightarrow M$ be defined by $T(h)=P(f h)$. We have $\|T\| \leq$ $\|f\|_{\infty} \leq 1$, and $T(1)=P(f)=a_{1}+a_{q} q^{-s}, T\left(q^{-s}\right)=P\left(q^{-s} f\right)=a_{1} q^{-s}$, so that the matrix of $T$ in the orthonormal basis $\left(1, q^{-s}\right)$ is

$$
\left(\begin{array}{cc}
a_{1} & 0 \\
a_{q} & a_{1}
\end{array}\right) .
$$

Now, it is well known ([24]) that such a matrix defines a contraction of $M$ if and only if $\left|a_{1}\right|^{2}+\left|a_{q}\right| \leq 1$, which proves the lemma.

Second proof of Lemma 2.1. We prove the following stronger result: 
Proposition 2.1. Let $f(s)=\sum_{n=1}^{\infty} a_{n} n^{-s} \in \mathcal{H}^{\infty}$ with $\|f\|_{\infty}=1$. Then

$$
\forall k \geq 1, \quad\left(\sum_{n \geq 2, \Omega(n)=k}\left|a_{n}\right|^{2}\right)^{1 / 2} \leq 1-\left|a_{1}\right|^{2} .
$$

Proof. We first show that we can assume $a_{n}=0$ for large $n$. In fact, if $N_{0} \geq 1$ and $\varepsilon>0$, it follows from Bohr's theorem ([11], [4] or [5]) that the series $\sum_{n=1}^{\infty} a_{n} n^{-\varepsilon} n^{-s}$ is uniformly convergent in $\mathbb{C}_{+}$, so that we can find an $N=N(\varepsilon)>N_{0}$ such that $\left\|\sum_{n=N+1}^{\infty} a_{n} n^{-\varepsilon} n^{-s}\right\|_{\infty} \leq \varepsilon$, and therefore $\left\|\sum_{n=1}^{N} a_{n} n^{-\varepsilon} n^{-s}\right\|_{\infty} \leq 1+\varepsilon$. If the finitely supported case has been settled, we get

$$
\left(\sum_{2 \leq n \leq N_{0}, \Omega(n)=k} \frac{\left|a_{n}\right|^{2}}{n^{2 \varepsilon}}\right)^{1 / 2} \leq\left(\sum_{2 \leq n \leq N, \Omega(n)=k} \frac{\left|a_{n}\right|^{2}}{n^{2 \varepsilon}}\right)^{1 / 2} \leq(1+\varepsilon)\left(1-\left|a_{1}\right|^{2}\right) .
$$

This is true for all $\varepsilon$, so by letting $\varepsilon \rightarrow 0$ we obtain

$$
\left(\sum_{2 \leq n \leq N_{0}, \Omega(n)=k}\left|a_{n}\right|^{2}\right)^{1 / 2} \leq 1-\left|a_{1}\right|^{2}
$$

Since this is true for all $N_{0} \geq 1$, this ends the proof of the lemma.

For a finitely supported sequence $\left(a_{n}\right)_{1 \leq n \leq N}$, let $r \geq 1$ be such that the prime factors of every $n \in[1, N]$ are in the set $\left\{p_{1}, \ldots, p_{r}\right\}$ of the $r$ first prime numbers. Using Bohr's theory, we pass to the polynomial of $r$ variables attached to $f$ :

$$
F\left(z_{1}, \ldots, z_{r}\right):=\sum_{\alpha \in \mathbb{N}^{r}} c_{\alpha} z_{1}^{\alpha_{1}} \cdots z_{r}^{\alpha_{r}}
$$

with $c_{\alpha}:=a_{n}$ if $n=p_{1}^{\alpha_{1}} \cdots p_{r}^{\alpha_{r}} \in[1, N]$ and $c_{\alpha}:=0$ otherwise, so that $F\left(p_{1}^{-s}, \cdots, p_{r}^{-s}\right)=f(s)$. Boas and Khavinson showed in [9] that for every $k \geq 1$ we have

$$
\left(\sum_{|\alpha|=k}\left|c_{\alpha}\right|^{2}\right)^{1 / 2} \leq 1-\left|c_{0}\right|^{2}=1-\left|a_{1}\right|^{2},
$$

where $|\alpha|=\alpha_{1}+\cdots+\alpha_{r}$. This ends the proof since

$$
\sum_{|\alpha|=k}\left|c_{\alpha}\right|^{2}=\sum_{n \geq 2, \Omega(n)=k}\left|a_{n}\right|^{2}
$$

Observe now the following consequence of the method of the second proof:

Proposition 2.2. If $f(s)=\sum_{n=1}^{\infty} a_{n} n^{-s} \in \mathcal{H}^{\infty}$ with $\|f\|_{\infty}=1$, then

$$
\sum_{p}\left|a_{p}\right| \leq 1-\left|a_{1}\right|^{2}
$$


Proof. As before, we can assume without loss of generality that $a_{n}=0$ for $n$ large. For a finitely supported sequence $\left(a_{n}\right)_{n \geq 1}$, we use Bohr's point of view, passing to

$$
F(z):=a_{1}+\sum_{k=1}^{\infty} f_{k}(z)
$$

where $f_{k}(z):=\sum_{\Omega(n)=k} a_{n} z^{\alpha(n)}$, and where $n=p_{1}^{\alpha_{1}} \cdots p_{r}^{\alpha_{r}}$ is the expansion of $n$ into prime factors and $z^{\alpha(n)}:=z_{1}^{\alpha_{1}} \cdots z_{r}^{\alpha_{r}}$ if $z=\left(z_{1}, z_{2}, \ldots\right)$. Fix $z$ in the unit infinite-dimensional polydisc $\left(\sup _{j \geq 1}\left|z_{j}\right| \leq 1\right)$, and consider, for $w \in \mathbb{D}$,

$$
g(w):=F(w z)=a_{1}+\sum_{k=1}^{\infty} f_{k}(z) w^{k} .
$$

Then, by Bohr's theory ([25]), we have $\|g\|_{\infty} \leq\|F\|_{\infty}=\|f\|_{\infty}=1$, and by Wiener's result ([24]), we have $\left|f_{k}(z)\right| \leq 1-\left|a_{1}\right|^{2}$ for $k \geq 1$.

For $k=1$, this gives $\left|\sum_{p} a_{p} z_{p}\right| \leq 1-\left|a_{1}\right|^{2}$. Optimizing with respect to $z=\left(z_{p}\right)_{p \text { prime }}$, we get the result, which can be viewed as an improvement of Bohr's inequality: $\sum_{p}\left|a_{p}\right| \leq\|f\|_{\infty}$ ([25] or [26]).

REMARK 2.1. Note that the inequalities (5) and (6) of the proof of Theorem 1.4 can be improved with the same method: if $\|f\|_{\infty}=1$, then

$$
\begin{aligned}
\sum_{p}\left(\sum_{q: q \geq p}\left|a_{p q}\right|^{2}\right)^{1 / 2} & \leq \frac{3 \sqrt{3}}{2 \sqrt{\pi}}\left(1-\left|a_{1}\right|^{2}\right), \\
\sum_{q}\left(\sum_{p: p \leq q}\left|a_{p q}\right|^{2}\right)^{1 / 2} & \leq \frac{3 \sqrt{3}}{2 \sqrt{\pi}}\left(1-\left|a_{1}\right|^{2}\right) .
\end{aligned}
$$

We shall use this improvement in the proof of Theorem 2.1, our next result.

THEOREM 2.1.

1) $\varrho_{1}\left(\mathcal{H}_{1}^{\infty}\right)=0$.

2) $\varrho_{1}\left(\mathcal{H}_{2}^{\infty}\right) \leq \sigma_{0}$, where $\sigma_{0}$ is the root of the equation

$$
2^{1-\sigma}+\frac{3 \sqrt{3}}{\sqrt{\pi}} \sqrt{F(4 \sigma)}=1,
$$

where $F$ is the function defined by $F(s):=\sum_{p} p^{-s}$. Moreover, 1.5903 $<\sigma_{0}<1.5904$.

3) $\varrho_{1}\left(\mathcal{H}^{\infty}\right) \leq \sigma_{1}$, where $\sigma_{1}$ is the root of the equation

$$
2^{-\sigma}+\frac{3 \sqrt{3}}{2 \sqrt{\pi}} \sqrt{F(4 \sigma)}+\sum_{n>1, \Omega(n) \geq 3} n^{-\sigma}=\frac{1}{2},
$$

where $F$ is defined as in 2). Moreover,

$$
\varrho_{1}\left(\mathcal{H}^{\infty}\right) \geq \log 3 / \log 2=1.5850 \ldots
$$


and $1.8153<\sigma_{1}<1.8154$. So if $f(s)=\sum_{n=1}^{\infty} a_{n} n^{-s} \in \mathcal{H}^{\infty}$, then $\sum_{n=1}^{\infty}\left|a_{n}\right| n^{-2} \leq\|f\|_{\infty}$.

Proof. 1) This is simply a reformulation of Bohr's inequality $\sum_{p}\left|a_{p}\right|$ $\leq\|f\|_{\infty}$.

2) Let

$$
f(s)=a_{1}+\sum_{p} a_{p} p^{-s}+\sum_{p, q: p \leq q} a_{p q}(p q)^{-s} \in \mathcal{H}_{2}^{\infty},
$$

with $\|f\|_{\infty}=1$. By an inequality of R. Blei ([8]), improving an inequality of Littlewood, we have

$$
\left(\sum_{p, q: p \leq q}\left|a_{p q}\right|^{4 / 3}\right)^{3 / 4} \leq\left[\sum_{p}\left(\sum_{q: q \geq p}\left|a_{p q}\right|^{2}\right)^{1 / 2}\right]^{1 / 2}\left[\sum_{q}\left(\sum_{p: p \leq q}\left|a_{p q}\right|^{2}\right)^{1 / 2}\right]^{1 / 2} .
$$

It follows, using (8) of Remark 2.1, that

$$
\left(\sum_{p, q: p \leq q}\left|a_{p q}\right|^{4 / 3}\right)^{3 / 4} \leq \frac{3 \sqrt{3}}{2 \sqrt{\pi}}\left(1-\left|a_{1}\right|^{2}\right)
$$

So, using Hölder's inequality and Proposition 2.2 we obtain

$$
\begin{aligned}
\sum_{n=1}^{\infty} \frac{\left|a_{n}\right|}{n^{\sigma}} & \leq\left|a_{1}\right|+\sum_{p} \frac{\left|a_{p}\right|}{2^{\sigma}}+\left(\sum_{p, q: p \leq q}\left|a_{p q}\right|^{4 / 3}\right)^{3 / 4}\left(\sum_{p, q: p \leq q} \frac{1}{(p q)^{4 \sigma}}\right)^{1 / 4} \\
& \leq\left|a_{1}\right|+\frac{1-\left|a_{1}\right|^{2}}{2^{\sigma}}+\frac{3 \sqrt{3}}{2 \sqrt{\pi}}\left(1-\left|a_{1}\right|^{2}\right) \sqrt{F(4 \sigma)}
\end{aligned}
$$

which is less than 1 if $2^{1-\sigma}+2 \cdot 3 \sqrt{3} \cdot \sqrt{F(4 \sigma)} /(2 \sqrt{\pi}) \leq 1$, that is, $\sigma \geq \sigma_{0}$, which ends the proof of 2 ).

3) First let $\sigma_{0}<\log 3 / \log 2$. Then $2^{-\sigma_{0}}>1 / 3$, and by Bohr's result we know that there exists a power series $f(z)=\sum_{n=0}^{\infty} a_{n} z^{n}$ with $|f(z)| \leq 1$ for $z \in \mathbb{D}$ and $\sum_{n=0}^{\infty}\left|a_{n}\right|\left(2^{-\sigma_{0}}\right)^{n}>1$. Let $F(s):=f\left(2^{-s}\right)=\sum_{n=0}^{\infty} a_{n}\left(2^{n}\right)^{-s}$. Then $F \in \mathcal{H}^{\infty},\|F\|_{\infty} \leq 1$, and yet

$$
\sum_{n=0}^{\infty}\left|a_{n}\right|\left(2^{n}\right)^{-\sigma_{0}}=\sum_{n=0}^{\infty}\left|a_{n}\right|\left(2^{-\sigma_{0}}\right)^{n}>1 .
$$

This shows that $\sigma_{0}$ is not an admissible abscissa and proves that $\varrho_{1}\left(\mathcal{H}^{\infty}\right) \geq$ $\log 3 / \log 2$.

Let now $f(s)=\sum_{n=1}^{\infty} a_{n} n^{-s} \in \mathcal{H}^{\infty}$ with $\|f\|_{\infty}=1$, and let $\sigma>0$. Then by 2) we know that

$$
\sum_{n \geq 1, \Omega(n) \leq 2} \frac{\left|a_{n}\right|}{n^{\sigma}} \leq\left|a_{1}\right|+\frac{1-\left|a_{1}\right|^{2}}{2^{\sigma}}+\frac{3 \sqrt{3}}{2 \sqrt{\pi}}\left(1-\left|a_{1}\right|^{2}\right) \sqrt{F(4 \sigma)} .
$$


So, using Lemma 2.1, we get

$$
\begin{gathered}
\sum_{n=1}^{\infty} \frac{\left|a_{n}\right|}{n^{\sigma} \leq}\left|a_{1}\right|+\frac{1-\left|a_{1}\right|^{2}}{2^{\sigma}}+\frac{3 \sqrt{3}}{2 \sqrt{\pi}}\left(1-\left|a_{1}\right|^{2}\right) \sqrt{F(4 \sigma)} \\
+\left(1-\left|a_{1}\right|^{2}\right)\left(\sum_{n>1, \Omega(n) \geq 3} n^{-\sigma}\right),
\end{gathered}
$$

which is less than 1 if $2^{-\sigma}+3 \sqrt{3} \cdot \sqrt{F(4 \sigma)} /(2 \sqrt{\pi})+\sum_{n>1, \Omega(n) \geq 3} n^{-\sigma} \leq 1 / 2$, that is, $\sigma \geq \sigma_{1}$. This ends the proof of the theorem.

REMARK 2.2. A result similar to 3) of Theorem 2.1 was proved for power series in [3], where the authors make use of the function $G(r):=\sum_{p} r^{p}$ (Lemma 2.1, p. 609).

We now examine the effect of taking $a_{1}=0$, or of replacing $\left|a_{1}\right|$ by $\left|a_{1}\right|^{2}$.

Proposition 2.3.

1) Let $f(s)=\sum_{n=2}^{\infty} a_{n} n^{-s} \in \mathcal{H}^{\infty}$. Then, if $\sigma_{2}:=\varrho / 2$, where $\zeta(\varrho)=2$ ( $\zeta$ being Riemann's zeta function), we have $\sum_{n=2}^{\infty}\left|a_{n}\right| n^{-\sigma_{2}} \leq\|f\|_{\infty}$. Moreover, $1.7286<\varrho<1.7287$ and $0.8643<\sigma_{2}<0.86435$.

2) If $\sum_{n=2}^{\infty}\left|b_{n}\right| n^{-\sigma} \leq\|g\|_{\infty}$ for all $g(s)=\sum_{n=2}^{\infty} b_{n} n^{-s} \in \mathcal{H}^{\infty}$, then $\sigma \geq 1 / 2$.

Proof. 1) The Cauchy-Schwarz inequality gives

$$
\begin{aligned}
\sum_{n=2}^{\infty} \frac{\left|a_{n}\right|}{n^{\sigma}} & \leq\left(\sum_{n=2}^{\infty}\left|a_{n}\right|^{2}\right)^{1 / 2}\left(\sum_{n=2}^{\infty} n^{-2 \sigma}\right)^{1 / 2} \\
& =\|f\|_{2}(\zeta(2 \sigma)-1)^{1 / 2} \leq\|f\|_{\infty}(\zeta(2 \sigma)-1)^{1 / 2} \leq\|f\|_{\infty}
\end{aligned}
$$

if $\zeta(2 \sigma)-1=1$, i.e. if $2 \sigma=\varrho$.

2) Let $f(z)=\sum_{n=1}^{\infty} a_{n} z^{n}$ be such that $|f(z)| \leq 1$ if $z \in \mathbb{D}$, and

$$
\sum_{n=1}^{\infty}\left|a_{n}\right| /(\sqrt{2})^{n}=\|f\|_{\infty}=1
$$

(e.g. $f(z)=z(1 / \sqrt{2}-z) /(1-z / \sqrt{2}))$. Let

$$
F(s):=f\left(2^{-s}\right)=\sum_{n=1}^{\infty} a_{n}\left(2^{n}\right)^{-s},
$$

and let $\sigma_{0}$ be defined by $2^{-\sigma_{0}}=1 / \sqrt{2}$, i.e. $\sigma_{0}=1 / 2$. We see that $\|F\|_{\infty} \leq 1$ and $\sum_{n=1}^{\infty}\left|a_{n}\right|\left(2^{n}\right)^{-\sigma_{0}}=\sum_{n=1}^{\infty}\left|a_{n}\right| /(\sqrt{2})^{n}=1$, so that we cannot go below $1 / 2$ with $\sigma$.

Proposition 2.4. Let $f(s)=\sum_{n=1}^{\infty} a_{n} n^{-s} \in \mathcal{H}^{\infty}$ with $\|f\|_{\infty}=1$. Then

where $\varrho$ is as in Proposition 2.3 .

$$
\left|a_{1}\right|^{2}+\sum_{n=2}^{\infty}\left|a_{n}\right| n^{-\varrho} \leq 1
$$


Proof. Using Lemma 2.1, we have

$$
\begin{aligned}
\left|a_{1}\right|^{2}+\sum_{n=2}^{\infty}\left|a_{n}\right| n^{-\varrho} & \leq\left|a_{1}\right|^{2}+\left(1-\left|a_{1}\right|^{2}\right) \sum_{n=2}^{\infty} n^{-\varrho} \\
& =\left|a_{1}\right|^{2}+\left(1-\left|a_{1}\right|^{2}\right)(\zeta(\varrho)-1) \\
& =\left|a_{1}\right|^{2}+\left(1-\left|a_{1}\right|^{2}\right)=1 . \text {. }
\end{aligned}
$$

Acknowledgments. Part of this work was done when the third-named author was visiting the Chennai Institute of Mathematics in March 2005.

\section{References}

[1] L. Aizenberg, Multidimensional analogues of Bohr's theorem on power series, Proc. Amer. Math. Soc. 128 (2000), 1147-1155.

[2] L. Aizenberg, I. B. Grossman and Yu. Korobeĭnik, Some remarks on Bohr radius for power series, Izv. Vyssh. Uchebn. Zaved. Mat. 2002, no. 10, 3-7 (in Russian).

[3] L. Aizenberg and A. Vidras, On the Bohr radius for two classes of holomorphic functions, Siberian Math. J. 45 (2004), 606-617.

[4] F. Bayart, Opérateurs de composition sur des espaces de séries de Dirichlet et problèmes d'hypercyclicité simultanés, Thèse à l'Université des Sciences et Technologies de Lille, 2002.

[5] - - Hardy spaces of Dirichlet series and their composition operators, Monatsh. Math. 136 (2002), 203-236.

[6] C. Bénéteau, A. Dahlner and D. Khavinson, Remarks on the Bohr phenomenon, Comput. Methods Function Theory 4 (2004), 1-19.

[7] C. Bénéteau and B. Korenblum, Some coefficient estimates for $H^{p}$ functions, in: Complex Analysis and Dynamical Systems, Contemp. Math. 364, Amer. Math. Soc., 2004, 5-14.

[8] R. C. Blei, Fractional cartesian products of sets, Ann. Inst. Fourier (Grenoble) 29 (1979), no. 2, 79-105.

[9] H. P. Boas and D. Khavinson, Bohr's power series theorem in several variables, Proc. Amer. Math. Soc. 125 (1997), 2975-2979.

[10] H. F. Bohnenblust and E. Hille, On the absolute convergence of Dirichlet series, Ann. of Math. 2 (1931), 600-622.

[11] H. Bohr, Über die gleichmässige Konvergenz Dirichletscher Reihen, J. Reine Angew. Math. 143 (1913), 203-211.

[12] -, A theorem concerning power series, Proc. London Math. Soc. 13 (1914), 1-5.

[13] - Almost Periodic Functions, Chelsea, 1933.

[14] E. Bombieri and J. Bourgain, A remark on Bohr's inequality, Int. Math. Res. Not. 2004, no. 80, 4307-4330.

[15] B. J. Cole and T. W. Gamelin, Representing measures and Hardy spaces for the infinite polydisk algebra, Proc. London Math. Soc. 53 (1986), 112-142.

[16] A. Defant, D. Garcia and M. Maestre, Bohr's power series theorem and local Banach space theory, J. Reine Angew. Math. 557 (2003), 173-197.

[17] P. G. Dixon, Banach algebras satisfying the non-unital non Neumann inequality, Bull. London Math. Soc. 27 (1995), 359-362.

[18] P. B. Djakov and M. S. Ramanujan, A remark on Bohr's theorem and its generalizations, J. Anal. 8 (2000), 65-77. 
[19] P. L. Duren, Theory of $H^{p}$ Spaces, Dover, 2000.

[20] H. Helson, Dirichlet Series, Regent Press, 2005.

[21] J.-P. Kahane, Some Random Series of Functions, 2nd ed., Cambridge Univ. Press, Cambridge, 1985.

[22] S. V. Konyagin and H. Queffélec, The translation 1/2 in the theory of Dirichlet series, Real Anal. Exchange 27 (2002), 155-175.

[23] D. Li et H. Queffélec, Introduction à l'étude des espaces de Banach : analyse et probabilités, Cours spécialisés 12, Soc. Math. France, 2004.

[24] V. I. Paulsen, G. Popescu and D. Singh, On Bohr's inequality, Proc. London Math. Soc. 85 (2002), 493-512.

[25] H. Queffélec, Harald Bohr's vision of Dirichlet series; old and new results, J. Anal. 3 (1995), 43-60.

[26] W. Rudin, Fourier Analysis on Groups, Wiley Classics Library, Wiley, 1990.

[27] J. Sawa, The best constant in the Khintchine inequality for complex Steinhaus variables, the case $p=1$, Studia Math. 81 (1985), 107-126.

[28] G. Tenenbaum, Introduction à la théorie analytique et probabiliste des nombres, Institut Élie Cartan, 1995.

[29] F. B. Weissler, Logarithmic Sobolev inequalities and hypercontractive estimates on the circle, J. Funct. Anal. 37 (1980), 218-234.

The Institute of Mathematical Sciences

Chennai 600 113, India

E-mail: balu@imsc.res.in

UFR de Mathématiques

Université de Lille 1

59655 Villeneuve d'Ascq Cedex, France

E-mail: Herve.Queffelec@math.univ-lille1.fr
Laboratoire de Mathématiques

Centre d'Orsay

Université Paris-Sud XI

Bâtiment 425

91405 Orsay, France

E-mail: bcalado999@aol.com

Received November 22, 2005

Revised version April 26, 2006 